\title{
A U.S. Survey of Sexual Harassment in Ophthalmology Training Using a Novel Standardized Scale
}

\author{
Brittni A. Scruggs, MD, PhD ${ }^{1}$ Lauren E. Hock, MD ${ }^{1}$ Michelle T. Cabrera, MD ${ }^{2,3}$ Kai Wang, $\mathrm{PhD}^{4}$ \\ Thomas A. Oetting, MS, MD ${ }^{1}$ Michael D. Abràmoff, MD, $\mathrm{PhD}^{1} \quad$ Erin M. Shriver, $\mathrm{MD}^{1}$ \\ ${ }^{1}$ Department of Ophthalmology and Visual Sciences, University of \\ lowa, lowa City, lowa \\ 2 Department of Ophthalmology, University of Washington, Seattle, \\ Washington \\ ${ }^{3}$ Department of Ophthalmology, Seattle Children's Hospital, Seattle, \\ Washington \\ ${ }^{4}$ Department of Biostatistics, College of Public Health, University of \\ lowa, lowa City, lowa \\ J Acad Ophthalmol 2020;12:e27-e35. \\ Address for correspondence Thomas A. Oetting, MS, MD, \\ Department of Ophthalmology and Visual Sciences, University of lowa \\ Hospitals and Clinics, 200 Hawkins Drive, lowa City, IA 52242 \\ (e-mail: thomas-oetting@uiowa.edu).
}

\begin{abstract}
Keywords

- ophthalmology training

- residency

- fellowship

- sexual harassment

Objective This study aimed to assess the frequency and severity of sexual harassment toward ophthalmology trainees.

Design Present study is an anonymous retrospective online survey.

Participants U.S. ophthalmology residents and fellows participated in this study. Methods Sexual harassment comments directed toward University of lowa ophthalmology trainees and faculty members were compiled. Statements were ranked by severity to develop the lowa Verbal Sexual Harassment Scale. A brief, anonymous online survey incorporating the scale was sent to all United States ophthalmology residency program directors to distribute among trainees. Participants rated the prevalence, severity, and frequency of verbal and physical sexual harassment during training.

Main Outcome Measures Response to the survey questions on the prevalence, severity, and frequency of reporting of verbal and physical sexual harassment in ophthalmology training.

Results Among 112 respondents (59 men and 53 women), 72 (64.3\%) experienced sexual harassment in the workplace from patients ( $86.8 \%$ of women vs. $44.1 \%$ of men; $p<0.0001,95 \%$ confidence interval $[\mathrm{Cl}]: 1.48-2.74)$. Trainees rarely experienced harassment by colleagues $(10.7 \%$ ) or supervisors $(8.9 \%)$. Women experienced more severe and frequent sexual harassment compared with men, with $54.7 \%$ women and $30.5 \%$ men experiencing sexual harassment weekly ( $p=0.013$, 95\% Cl: $1.29-5.71)$. Unwanted touching was the most common physical harassment type. The trainees' threshold for reporting sexual harassment was higher than their worst actual experience $(p<0.0001, F(2,282)=67.59)$. Few trainees formally reported verbal $(6.3 \%)$ or physical sexual harassment (1.8\%). Trainees most commonly responded to harassment by redirecting the harasser (67.9\%). Only $33.9 \%$ of trainees rated their institution's sexual harassment training as helpful preparation for addressing harassment.
\end{abstract}

received

October 24, 2019 accepted after revision January 21,2020
DOI https://doi.org/

10.1055/s-0040-1705092. ISSN 2475-4757.
Copyright $\odot 2020$ by Thieme Medical Publishers, Inc., 333 Seventh Avenue, New York, NY 10001, USA. Tel: +1(212) 760-0888.
License terms

()(1) $\Theta \circledast$ 
Conclusion Most ophthalmology trainees experienced sexual harassment with almost all harassment coming from patients. Female trainees reported substantially greater severity and frequency of sexual harassment. There remains an unmet need for targeted response training in ophthalmology training programs.

Workplace sexual harassment in medicine decreases job satisfaction and negatively impacts both personal confidence and professional career advancement. ${ }^{1,2}$ Such effects have the potential to decrease a physician's clinical performance and, thus, patient safety. Cabrera et al showed that $59 \%$ of mostly female ophthalmologists and ophthalmology trainees had experienced sexual harassment during their careers, most commonly while in training. ${ }^{3}$ Few had reported their most significant sexual harassment experiences to an authority, consistent with low-reporting rates in other fields. ${ }^{3}$ These studies highlight the need for academic institutions to better address sexual harassment among their trainees.

Subtle sexual harassment behaviors may not have been adequately examined in the Cabrera et al study. ${ }^{3}$ Chronic exposure to these subtle forms of harassment (i.e., microaggressions), including sexual objectification, reinforcement of traditional gender roles, and gender inferiority stereotypes, has been shown to increase perceived stress, ${ }^{4}$ depressive symptoms, ${ }^{4}$ hypertension, ${ }^{5}$ and poor sleep. ${ }^{5}$ A major hindrance to discussing the prevalence and pervasiveness of sexual harassment in the clinical setting is the lack of a standardized scale by which to measure its severity and to relate it to the physicians' effectiveness. A report from 1980 categorized sexual harassment behaviors into the following five types: (1) sexist remarks/behaviors; (2) inappropriate or offensive sexual advances; (3) solicitation of sexual activity; (4) coercion of sexual activity; and (5) sexual crimes and misdemeanors. ${ }^{6}$ This system has been used in numerous nonmedical and medical studies to assess sexual harassment. ${ }^{1}$ However, these categories neither address the nuances of subtle behaviors that frequently affect clinicians nor do they relate the harassment behaviors to a clinician's ability to perform his/her job, which may lead to patient harm.

Therefore, we have developed a standardized verbal sexual harassment scale by which physicians at any level of training can score sexual harassment comments and behaviors, both overt and subtle, that they experience in the workplace. This scale quantifies the effect that the harassment has on the physician's ability to perform clinical responsibilities at the highest level. The standardized scale was then introduced in a national survey distributed to ophthalmology trainees and used to assess the prevalence, severity, and reporting of sexual harassment experienced during ophthalmology training.

\section{Methods}

A cross-sectional anonymous online survey was sent to all U.S. ophthalmology program directors for voluntary distribution to their trainees. Participants rated their experiences with sexual harassment during ophthalmology training using a novel standardized scale developed and validated at a single academic center. Institutional Review Board (IRB) approval for scale development and survey distribution was obtained at the University of Iowa Hospitals and Clinics (UIHC) prior to study initiation.

\section{Development of a Standardized Sexual Harassment Scale}

In May 2017, all female ophthalmology residents and female faculty at UIHC Department of Ophthalmology and Visual Sciences were asked to voluntarily document sexual harassment comments they had received from patients, colleagues, and/or faculty during the 2016 to 2017 academic year. Eleven residents (100\%) and five faculty members (71\%) anonymously submitted comments. All female residents and faculty responded that they had experienced adverse comments. All comments were categorized as sexual harassment as defined by the U.S. Equal Employment Opportunity Commission (EEOC, https://www.eeoc.gov/eeoc/publications/ $f s$-sex.cfm): "unwelcome sexual advances, requests for sexual favors, and other verbal or physical conduct of a sexual nature," when this conduct "explicitly or implicitly affects an individual's employment, unreasonably interferes with an individual's work performance, or creates an intimidating, hostile, or offensive work environment ${ }^{7}$."

These anonymous comments were compiled and sent to all female ophthalmology residents of the 2017 to 2021 graduating classes $(N=9)$ for scoring according to how it affected their clinical performance. Similar statements were intentionally included to determine variability across scorers. The residents were asked to voluntarily fill in a score 1 to 5 for each comment, where " 1 " signifying a statement that did not affect their actions, self-respect, or ability to practice. A score of " 5 " indicated that the statement made it impossible for the resident to provide the highest level of care. Averaged numerical scores \pm standard error of the mean (SEM) were assigned to each comment, and the ranked statements were then analyzed for general themes to develop a five-point Likert's scale of verbal sexual harassment categories, the Iowa Verbal Sexual Harassment Scale (-Fig. 1).

\section{Distribution of an Anonymous Online Survey}

Once the Iowa Verbal Sexual Harassment Scale was developed with various categories of verbal sexual harassment levels, a 15-question Qualtrics survey (-Supplementary Material) was created to query ophthalmology trainees about their experiences with sexual harassment in residency and/or fellowship. The residency program director at the Department of Ophthalmology and Visual Sciences (T.A.O.) at University of Iowa sent an e-mail with the survey link and 


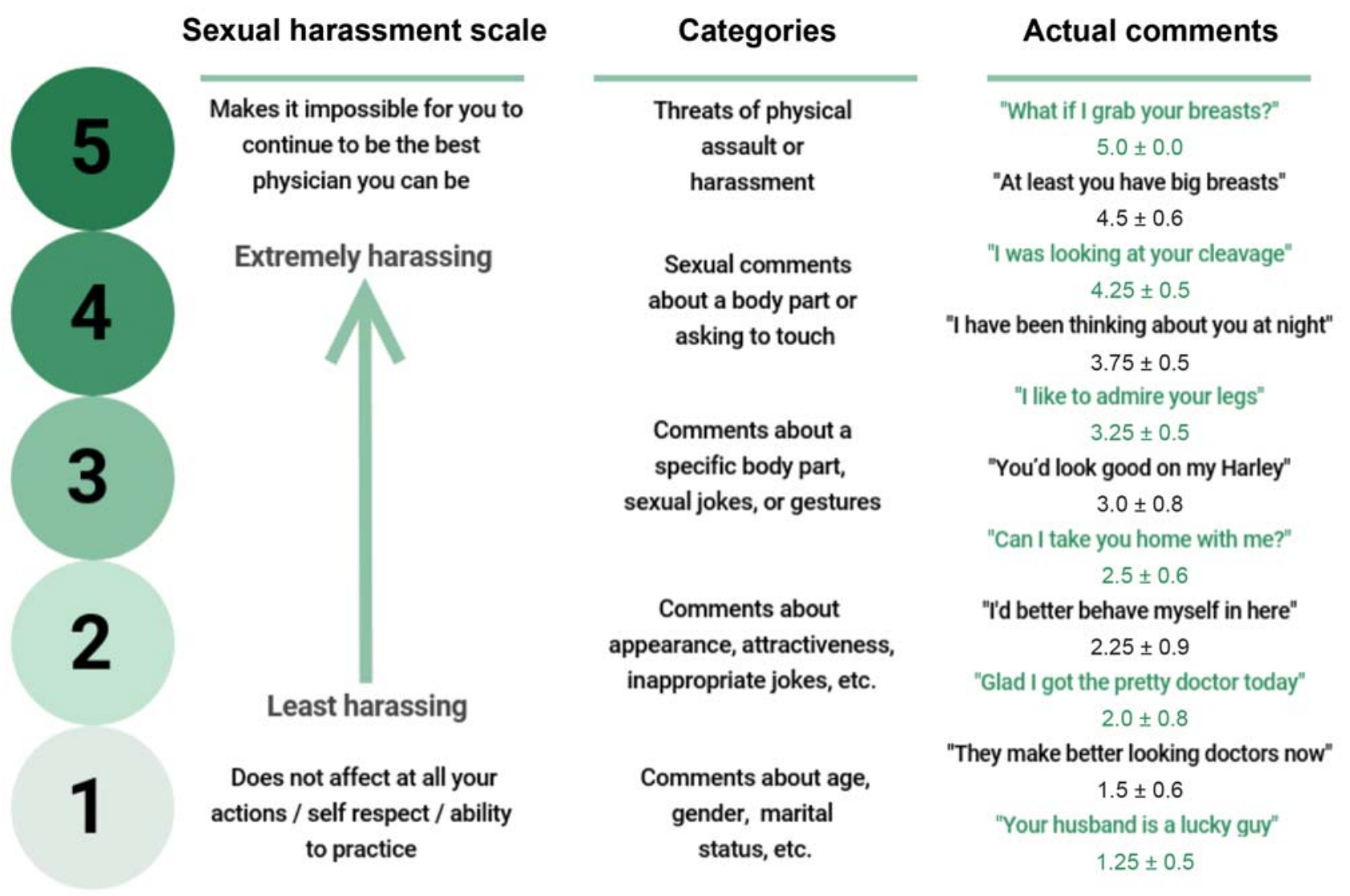

Fig. 1 The lowa Verbal Sexual Harassment Scale. Comments were compiled anonymously and scored by the $2017-2021$ graduating classes of female ophthalmology residents. A score of “ 1 ” signifies a statement that did not affect a trainee's actions, self-respect, or ability to practice. A score of " 5 " indicated that it was impossible for the resident to provide the highest level of care. Averaged numerical scores were assigned to each comment, and the ranked statements were then analyzed for general themes to develop five sexual harassment scores.

study information to all 120 United States program directors via the Association of University Professors of Ophthalmology Listserv; the program directors could choose to distribute the survey to their trainees, including residents and fellows. To preserve anonymity for programs and respondents, tracking information regarding which program directors chose to distribute the survey to their trainees was not obtained. Any ophthalmology trainee who received the survey link could choose to respond. Resources were readily available as attachments to the distributed e-mail in the event that a participant needed to discuss or report his/her sexual harassment experiences.

All survey participants voluntarily completed the survey (3-5 minutes) by clicking on the distributed link to the Ulowa Qualtrics survey (Supplemental Material). The 15 questions were all optional. Participants reported their self-identified gender. The survey queried the trainee's experience with verbal (i.e., oral or written) or physical sexual harassment in the workplace during ophthalmology training, gathering information regarding the frequency and severity of the harassment. Specifically, the participant identified their harassers during training as patients, staff member/colleagues, and/or supervising faculty (-Fig. 2). Participants reported both their highest experienced score on the Iowa Verbal Sexual Harassment Scale in the previous
12 months and also over their entire training program (-Fig. 3). Each participant identified the score they deemed their "reporting threshold" (-Fig. 3). The participants also reported the frequency at which they experienced each severity score; they were given the options of never, every 2 to 5 years, annually, monthly, and weekly (-Fig. 4).

To assess trainees' experiences with more severe forms of sexual harassment, various acts of physical harassment or blackmail/coercion were listed, and the trainee could choose none or all that applied to their experiences (-Table 1). The trainees were queried regarding strategies they had used to respond to harassment behavior in the workplace (-Table 2 ); the trainee could choose as many of the 11 strategies listed that applied or he/she could write in a unique response. All ACGME (Accreditation Council for Graduate Medical Education)-accredited training programs are required to have written policies covering sexual harassment. A five-point Likert's scale was used to assess the helpfulness of institutional training in preparing the trainee to speak directly with a harasser about their behavior in the moment (-Fig. 5). The trainees' experience with reporting verbal and/or physical sexual harassment during training was assessed in addition to their knowledge of the protocols for prompt reporting and any factors that contributed to them not reporting sexual harassment (-Fig. 6). 


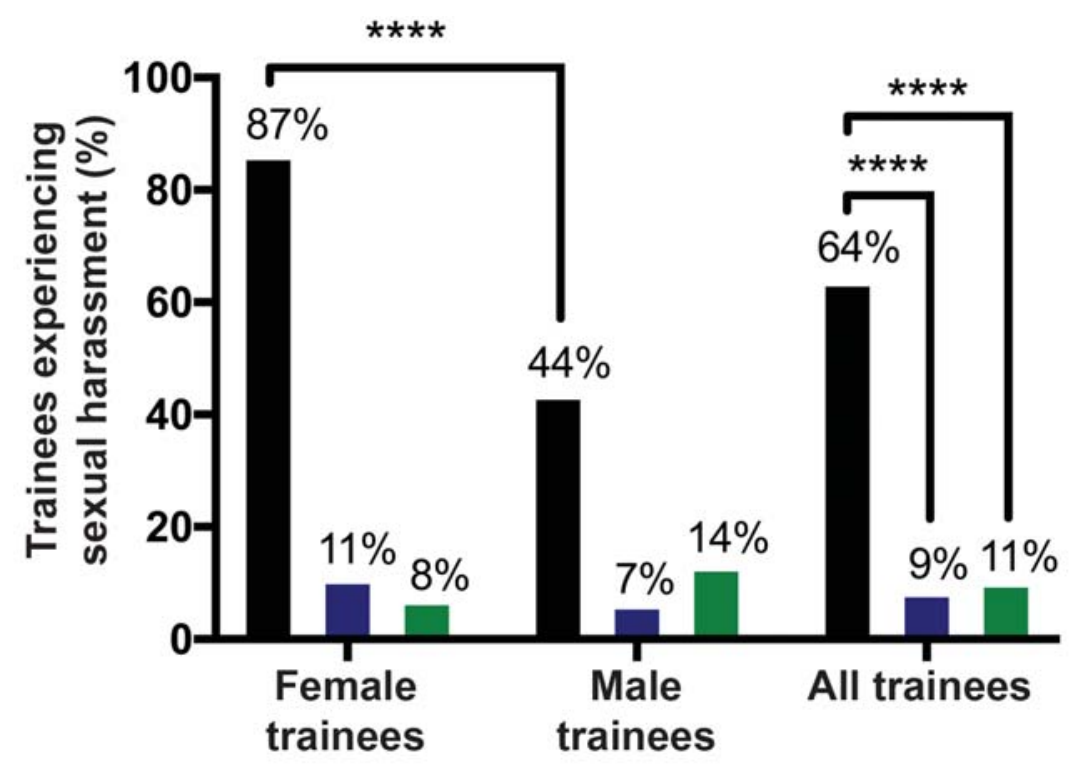

- Patients Faculty Colleague/Staff members

Fig. 2 Verbal and physical sexual harassment by patients (black), faculty (blue), or colleagues (green) directed toward female and male ophthalmology trainees. Female $(n=53)$ and male $(n=59)$ trainees experienced the most sexual harassment from patients, and women experienced patient-initiated sexual harassment more frequently than men as determined using Fisher's exact test; $\left.{ }^{* * * *}\right)$ signifies $p<0.0001$.

Female vs. male trainees

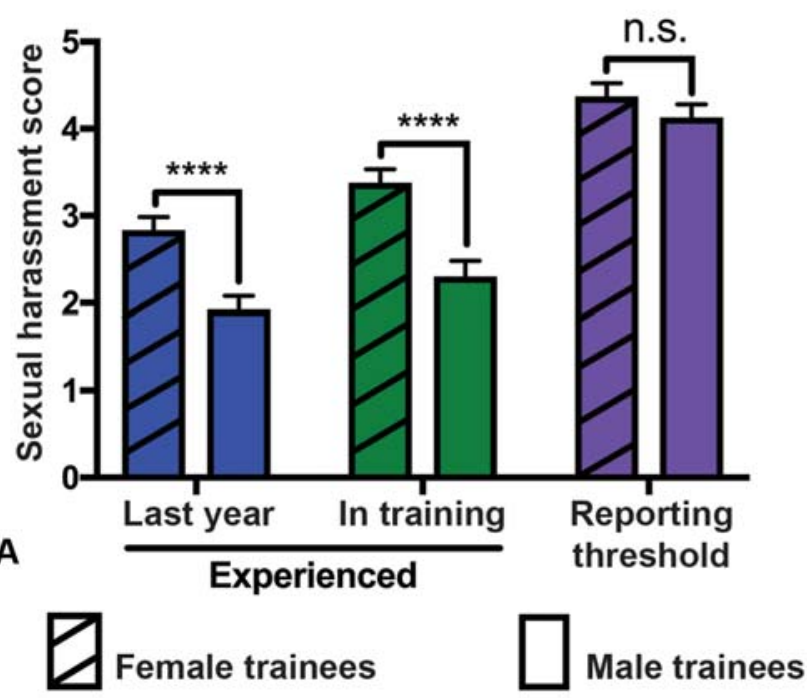

All trainees

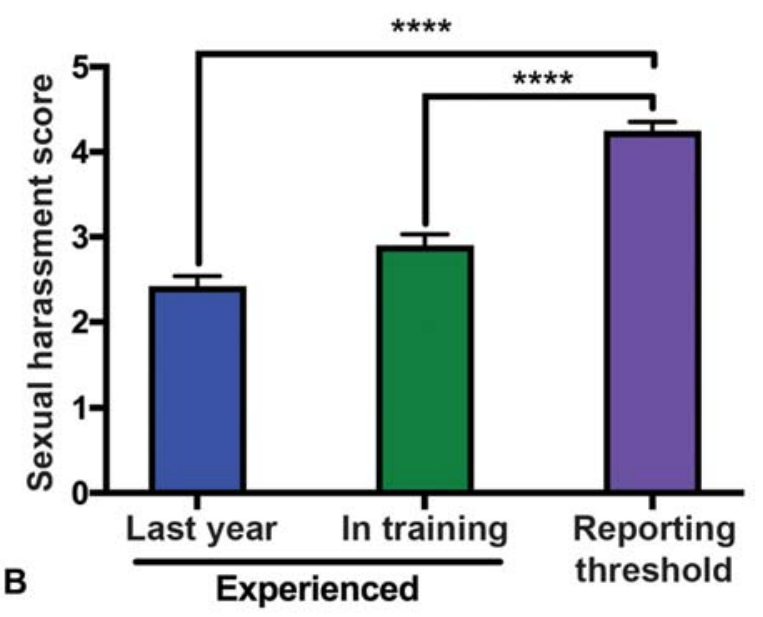

Fig. 3 Highest severity of verbal sexual harassment experienced by female and male ophthalmology trainees compared with their threshold for reporting.(A) Based on the lowa Verbal Sexual Harassment Scale, female trainees experienced greater severity of sexual harassment compared with male trainees both in the last year and over their entire training period. Both men and women had similar reporting threshold scores. (B) Among ophthalmology trainees of both genders, the score designated as the reporting threshold was significantly greater than the worst verbal sexual harassment they had experienced. $\left.{ }^{* * * *}\right)$ signifies $p<0.0001$; n.s., no significance.

\section{Participants}

The resulting Iowa Verbal Sexual Harassment Scale was applied to a national survey of ophthalmologists in training (e.g., residents or fellows) who, at least part of the year, worked in the United States in 2019. Institutional Review Board (IRB) approval for this study was obtained at the UIHC. Research adhered to the tenets of the Declaration of Helsinki.

\section{Statistical Analyses}

The Iowa Verbal Sexual Harassment Scale reported scores were averaged for all participants. Prevalence was calculated by assuming that an average score greater than zero indicated the presence of sexual harassment for each participant, and Fisher's exact test was used to test for differences between female and male trainees. Statistical analyses with a two-tailed 


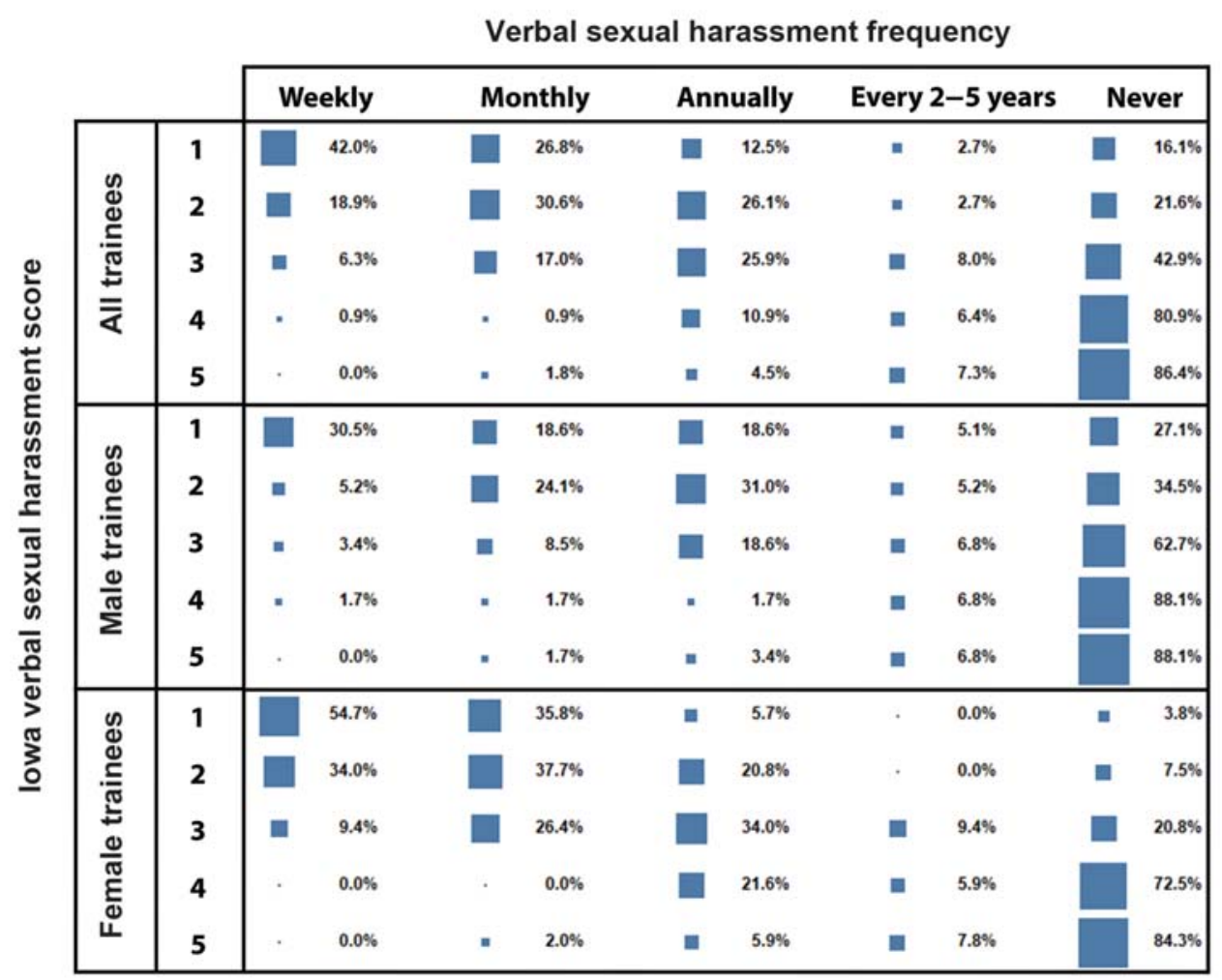

Fig. 4 The frequency of verbal sexual harassment experienced by female and male ophthalmology trainees. Using the lowa Verbal Sexual Harassment Scale, female $(n=53)$ and male $(n=59)$ ophthalmology trainees reported how frequently they experience each sexual harassment score. For each score, the percentages across each row have a sum of 100\%. A score of "1" signifies a statement that did not affect a trainee's actions, self-respect, or ability to practice. A score of " 5 " indicates a statement that made it impossible for the trainee to provide the highest level of care.

Table 1 Acts of physical harassment or blackmail/coercion experienced in ophthalmology training

\begin{tabular}{|c|c|c|c|}
\hline & $\begin{array}{l}\text { Female } \\
\text { trainees } \\
n(\%)^{a}\end{array}$ & $\begin{array}{l}\text { Male } \\
\text { trainees } \\
n(\%)^{a}\end{array}$ & $p$-Value ${ }^{b}$ \\
\hline None & $40(75.5)$ & $54(91.5)$ & 0.037 \\
\hline Blackmail or coercion & $0(0)$ & $1(1.7)$ & $>0.9999$ \\
\hline $\begin{array}{l}\text { Frequent or unwanted touching/massage of neutral body parts, } \\
\text { such as shoulder, back, or low back }\end{array}$ & $11(20.8)$ & $3(5.1)$ & 0.02 \\
\hline Sexual assault involving touching sensitive body parts, such as waist or upper thigh & $1(1.9)$ & $1(1.7)$ & $>0.9999$ \\
\hline Sexual assault involving touching buttocks or forced kissing & $1(1.9)$ & $1(1.7)$ & $>0.9999$ \\
\hline Sexual assault involving touching breasts or genitals or rubbing groin on trainee & 1 (1.9) & $1(1.7)$ & $>0.9999$ \\
\hline
\end{tabular}

${ }^{a}$ Trainees could report more than one behavior.

${ }^{\mathrm{b}}$ Significance was defined by $p<0.05$.

unpaired $t$-test and Welch's correction was used for comparison of the experienced scores by female and male ophthalmology trainees. Comparisons of reporting threshold scores and the highest experienced scores in the last year and ever in training were performed using one-way analysis of variance (ANOVA) followed by pairwise comparisons of the groups using Dunnett's testing. Data regarding sexual harassment prevalence and strategies used to respond to sexual harassment behavior were both analyzed by Pearson's Chi-square test then Fisher's exact test to determine differences among female and male trainees. Significance for comparisons was defined as $p<0.05$ for all analyses. All statistical tests were performed using GraphPad Prism 4.0b for Macintosh (GraphPad Software, San Diego, CA), and all values were reported as mean \pm SEM. Tableau Software (Seattle, WA) and GraphPad Prism 4.0b for Macintosh were used to generate the figures.

\section{Results}

\section{lowa Verbal Sexual Harassment Scale}

A total of 43 comments were submitted by 11 residents and 5 faculty members and then ranked by all female ophthalmology residents $(n=9)$ in the 2017 to 2018 academic year according to how it affected their clinical performance, as described 
e32 Sexual Harassment in Ophthalmology Training Scruggs et al.

Table 2 Strategies ophthalmology trainees used to respond to sexual harassment behavior in the workplace

\begin{tabular}{|c|c|c|c|}
\hline & $\begin{array}{l}\text { Female } \\
\text { trainees } \\
n(\%)^{a}\end{array}$ & $\begin{array}{l}\text { Male } \\
\text { trainees } \\
n(\%)^{a}\end{array}$ & $p$-Value ${ }^{b}$ \\
\hline Not applicable & $8(15.0)$ & $23(38.9)$ & 0.006 \\
\hline Redirect the encounter & $42(79.2)$ & $34(57.6)$ & 0.02 \\
\hline Joke about the behavior & $18(34.0)$ & $10(16.9)$ & 0.05 \\
\hline Avoid the harasser & $24(45.3)$ & $5(8.4)$ & $<0.0001$ \\
\hline Modify attire/demeanor & $10(18.9)$ & $0(0)$ & 0.0003 \\
\hline Limit personal information sharing, including online presence & $15(28.3)$ & $13(22.0)$ & 0.51 \\
\hline Enlist a chaperone for future encounters with harasser & $18(34.0)$ & $1(1.7)$ & $<0.0001$ \\
\hline Speak directly with the harasser about their behavior & $12(22.6)$ & $7(11.9)$ & 0.14 \\
\hline Criticize the harasser, express disgust, and/or verbally threaten the harasser & $3(5.7)$ & $0(0)$ & 0.10 \\
\hline Document the harassment behavior without reporting it (e.g., in a patient chart) & $4(7.5)$ & $1(1.7)$ & 0.19 \\
\hline
\end{tabular}

${ }^{\mathrm{a}}$ Trainees could report more than one behavior.

bSignificance was defined by $p<0.05$.

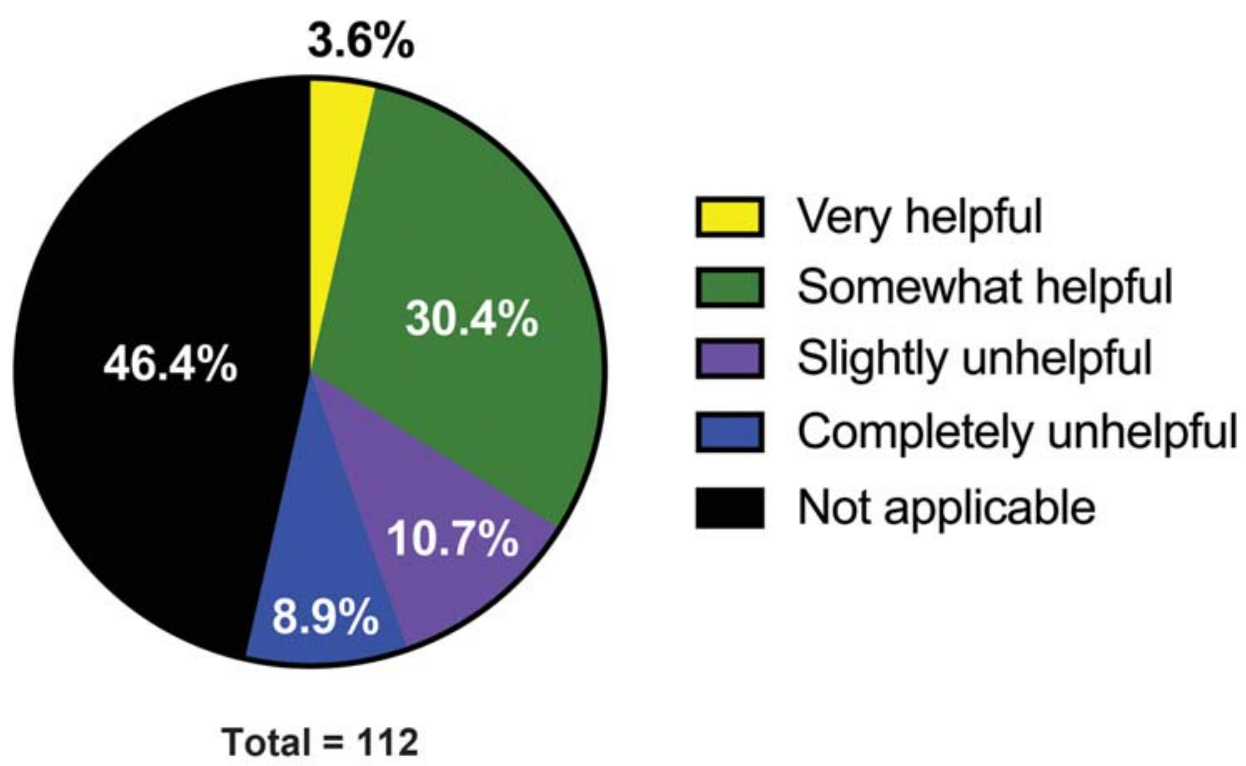

Fig. 5 Assessment of sexual harassment training programs by ophthalmology trainees. Trainees $(n=112)$ rated their institution's training program based on their perceived helpfulness in preparing the trainee to speak directly with a harasser in the moment. If trainees did not participate in a sexual harassment training program during their ophthalmology residency and/or fellowship, they were instructed to choose "not applicable."

previously. From these 43 comments, representative examples of patient-initiated sexual harassment directed toward ophthalmology residents with their averaged ranking \pm SEM included: "Your husband is a lucky guy" $(1.71 \pm 0.36)$; "I'd better behave myself in here" ( $2.42 \pm 0.43)$; "I like to admire your legs" ( $3.29 \pm 0.36)$; "I have been thinking of you at night" $(4.00 \pm 0.31)$; and "What if I grab your breasts" $(5.0 \pm 0.0)$. In developing the Iowa Verbal Sexual Harassment Scale ( - Fig. 1), analysis of the ranked comments revealed the following five categories, in increasing severity: (1) age, gender, and marital status; (2) appearance, attractiveness, and inappropriate jokes; (3) specific body parts, sexual jokes, or sexual gestures; (4) sexual comments regarding a body part or asking to touch; and (5) threats of physical assault or physical harassment.

\section{National Sexual Harassment Survey Results}

The Iowa Verbal Sexual Harassment Scale described above was used to query ophthalmology trainees nationally about sexual harassment in the workplace. At the time of the survey, the SF Match Residency and Fellowship Matching Services reported 484 ophthalmology residents and 338 ophthalmology fellows in the United States in 2018 to 2019. No information was received on which program directors distributed the survey link. The survey had 112 survey respondents (59 men, 52.6\%), all of whom were U.S. ophthalmology trainees as of March 2019. There were a total of 72 of 112 (64.3\%) ophthalmology trainees who experienced sexual harassment in the workplace by patients (score $>0$ ). As shown in - Fig. 2, female trainees $(46 / 53,86.8 \%)$ experienced patient-initiated sexual 


\section{Factors that contribute to trainees not reporting sexual harassment in the workplace}

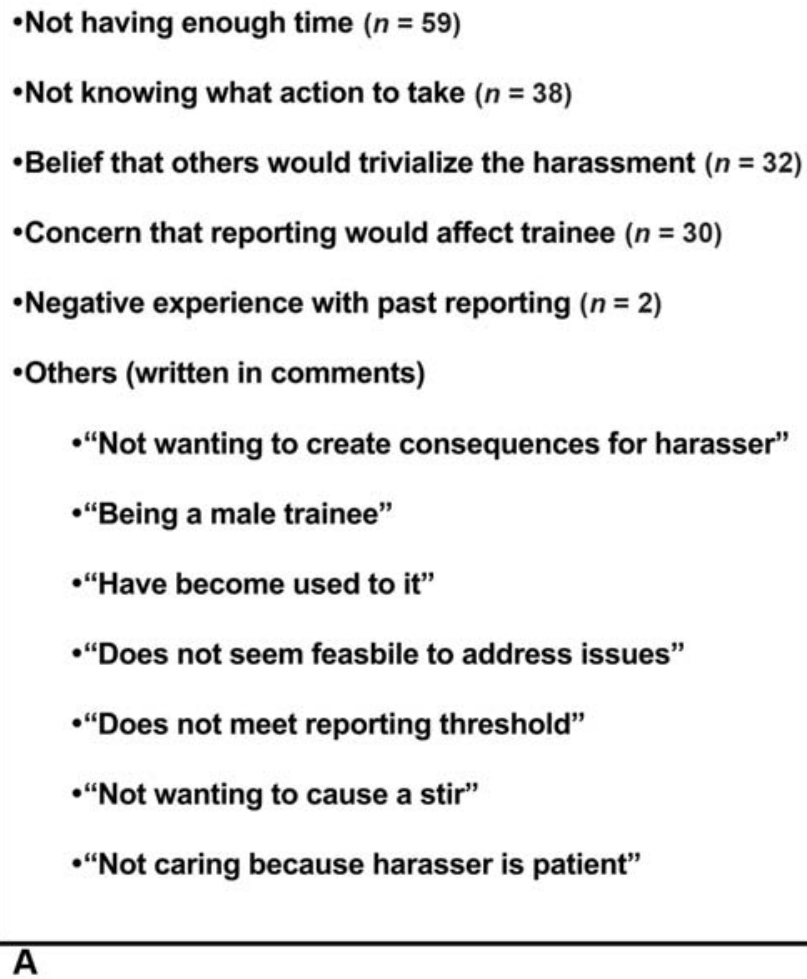

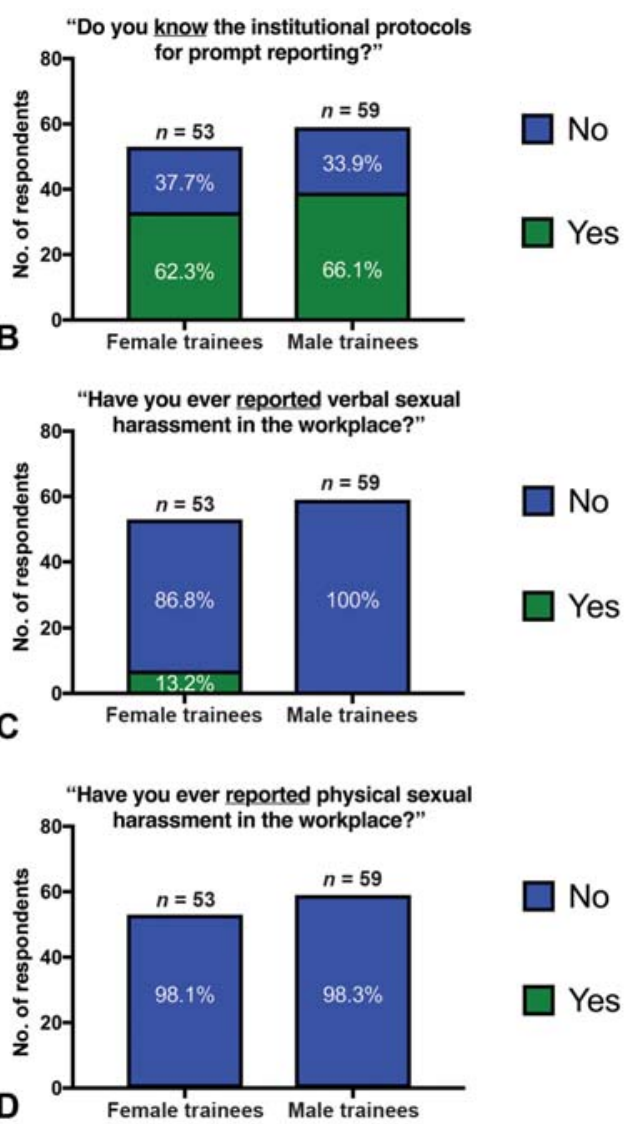

Fig. 6 Reporting of sexual harassment by ophthalmology trainees. (A) Ophthalmology trainees $(n=112)$ identified factors that limited reporting sexual harassment during the work day. (B) Trainees were asked whether or not they knew the protocols at their institution for prompt reporting of workplace sexual harassment. (C, D) The numbers of survey respondents who have ever reported verbal sexual harassment (C) or physical sexual harassment (D) were determined.

harassment more frequently than male trainees $(26 / 59$ or $44.1 \%$; odds ratio $[\mathrm{OR}]=8.34,95 \%$ confidence interval $[\mathrm{CI}]$ : $1.48-2.74, p<0.0001)$. Sexual harassment by patients occurred more frequently than harassment initiated by faculty (10/112, 8.9\%; OR = 18.36, 95\% CI: 8.56-36.9, $p<0.0001)$ or staff members or colleagues $(12 / 112,10.7 \%$; $\mathrm{OR}=15,95 \% \mathrm{CI}$ : 7.44-29.79, $p<0.0001$ ).

Female respondents experienced sexual harassment with increased severity compared with male respondents (-Fig. 3A). Using the Iowa Verbal Sexual Harassment Scale, female trainees rated their most severe sexual harassment during training as $3.38 \pm 0.15(n=50)$, whereas male trainees scored their most severe experience as $2.30 \pm 0.18(n=40$, $p<0.0001$ ). When asked what score would be considered "reportable" to them, there was no difference between female and male trainees with average scores of $4.37 \pm 0.15(n=51)$ and $4.13 \pm 0.15(n=54)$, respectively $(p=0.26)$. There was a significant difference between the reporting threshold scores and the highest experienced scores for all trainees whether in the last year or ever in training $(\mathrm{F}(2,282)=67.59, p<0.0001$; -Fig. 3B).

Female and male ophthalmology trainees also reported how frequently they experienced each sexual harassment score; they were given the options of never, every 2 to 5 years, annually, monthly, and weekly (-Fig. 4). Of all respondents, 51 of 53 (96.2\%) female trainees and 43 of 59 (72.9\%) male trainees had experienced comments regarding age, gender, or marital status (score of 1) during training, with 29 of 53 (54.7\%) female trainees experiencing a score of " 1 " on a weekly basis compared to 18 of 59 (30.5\%) men $(\mathrm{OR}=2.752,95 \% \mathrm{CI}: 1.29-5.71$, $p=0.013)$. A total of 18 of 53 (34.0\%) women reported weekly comments about appearance, attractiveness, or inappropriate jokes (score of 2 ) compared with 3 of 59 (5.2\%) men ( $O R=9.6$, 95\% CI: 2.67-32.01, $p=0.0002$ ). At least annually, 37 of 53 (69.8\%) female trainees and 18 of 59 (30.5\%) male trainees experienced comments about specific body parts and/or sexual gestures (score of 3; OR = 5.267, 95\% CI: 2.37-11.95, $p<$ $0.0001)$. Female respondents $(11 / 51,21.6 \%)$ were more likely than male respondents (3/59 or $5.1 \%)$ to experience sexual comments regarding a body part (score of 4 ) at least yearly $(\mathrm{OR}=4.89,95 \% \mathrm{CI}: 1.33-16.99, p=0.02)$. There was no significant difference in the rates that men $(7 / 59,11.9 \%)$ and women (8/51, 15.7\%) experienced extremely harassing comments during training that involved threats of physical sexual assault or harassment (score of 5; OR $=1.382,95 \% \mathrm{CI}$ : 0.4386-3.765, $p=0.59)$.

More female ( $13 / 53$ or $24.5 \%$ ) than male trainees (5/59 or $8.5 \%)$ reported physical harassment, blackmail, and/or coercion during ophthalmology training $(\mathrm{OR}=3.51,95 \% \mathrm{Cl}$ : 1.14-9.40, $p=0.037$ ). Unwanted touching or massage was 
the most common form of physical harassment experienced (-Table 1). Trainees most commonly responded to harassment behavior by redirecting the harasser, and female trainees were more likely than male trainees to avoid the harasser $(\mathrm{OR}=8.94,95 \% \mathrm{CI}: 3.03-22.89, p<0.0001)$, enlist a chaperone (OR $=29.83,95 \% \mathrm{CI}: 4.61-316.90, p<0.0001)$, or modify their attire $(\mathrm{OR}=$ infinity, 95\% CI: 3.745-infinity, $p=0.0003)$. Twelve of 53 (22.6\%) women and 7 of 59 (11.9\%) men had spoken directly with a harasser about their behavior (-Table 2).

Only 34\% of trainees rated their institution's sexual harassment training program as helpful in preparing them to address harassment (-Fig. 5). Thirty-eight of 112 (33.9\%) trainees "did not know what action to take" in the moment. Few trainees had ever reported verbal sexual harassment $(7 / 112,6.3 \%)$ or physical sexual harassment $(2 / 112,1.8 \%)$ to an authority. Reasons for not reporting included "lack of time" in 59 of $112(52.7 \%)$ respondents. In addition, 32 of 112 (28.6\%) trainees believed that "others would trivialize the harassment," 30 of 112 (26.8\%) trainees were concerned that reporting would affect them negatively, and 2 of $112(1.8 \%)$ had a prior negative reporting experience (-Fig. $\mathbf{6}$ ).

\section{Discussion}

This study developed a new standardized sexual harassment scale, known as the Iowa Verbal Sexual Harassment Scale, and used it to classify verbal harassment based on the degree of impact it has on a clinician's actions, self-respect, and/or ability to provide the highest level of care in the moment. This standardized scale, based on actual statements directed at current ophthalmology residents and faculty, allows a clear classification of highly prevalent harassment behaviors that affect the physician's ability to provide excellent patient care but do not meet typical thresholds for reporting. By providing a more granular sexual harassment classification system, data from this scale applied to a national survey highlight the breadth and severity of patient verbal harassment experienced by our trainees.

A meta-analysis found that sexual harassment was the most common form of abusive behavior in the U.S. medical training encountered by $36.2 \%$ of resident physicians. ${ }^{8}$ This study demonstrates that female trainees were more likely than male trainees to experience sexual harassment in ophthalmology training; this corroborates recent data showing that women experience more gender bias in academic medicine. ${ }^{1}$ More than half of women and nearly one-third of men experienced a score of "1" (e.g., "They make better looking doctors every day") on a weekly basis. The more severe harassing comments (e.g., "At least you have big breasts") also occurred with high frequency, with more than a quarter of female residents experiencing these comments during their training. Residents scored these severe comments as "making it impossible for (them) to continue to be the best physician (they) could be." Cabrera et al similarly reported that $87 \%$ of harassed female ophthalmologists had associated negative impacts on their professional lives, including interference with their ability to work. ${ }^{3}$ Altogether, these data suggest that both the frequency and severity of sexual harassment comments in ophthalmology training contribute to a suboptimal training and work environment.

Cabrera et al reported that academic attendings or faculty members were the most frequent harasser of ophthalmologists. $^{3}$ In contrast, this study shows that patient-initiated harassment was, by far, the most prevalent type of sexual harassment experienced in training. This may be related to the fact that the novel scoring system measures verbal sexual harassment, including more subtle behaviors. This study is the first to describe a high prevalence of patient-initiated sexual harassment in ophthalmology. Future endeavors include studying this subject and its ethical and medicolegal ramifications. Additionally, inclusion of different types of comments in the Iowa Verbal Sexual Harassment Scale further improves recall of respondents' experiences. While physical sexual harassment, blackmail, and coercion were not included on the verbal sexual harassment scale, they were investigated in the survey. These behaviors, like verbal sexual harassment, were more frequently experienced by women with unwanted touching reported by one-fifth of female trainees.

The survey data also suggest that trainees who are sexually harassed are ill-prepared to respond to physical or verbal harassment behavior. More than half of trainees redirect the harasser more often than addressing the behavior directly. Women were more likely than men to joke about the harassment, avoid the harasser, modify attire and/or demeanor, or enlist a chaperone for future encounters with the harasser. Only one-third of all trainees perceived that their institution's existing sexual harassment training was helpful in preparing them to speak directly with a harasser in the moment. Averaged among all trainees, the reporting threshold score was documented as being significantly greater than that of the most severe form of verbal harassment experienced during training. This finding suggests that the respondents consider their own experiences with verbal harassment as nonreportable, in spite of a high severity of experiences documented in this study. There were several barriers that contributed to trainees not addressing the harassment behavior, including lack of time, not knowing what action to take, and concern that they would be negatively affected or trivialized. These data suggest that sexual harassment training programs should provide tools for all medical trainees to address sexual harassment in the moment and to report harassment that may affect clinical care (e.g., serious offenses by faculty/colleagues/ patients and/or frequent offenders in spite of feedback). Training programs should emphasize that reporting is acceptable and expected while empowering trainees to respond to harassment in a way that does not decrease clinical efficiency.

These data may be used to address two principal unmet needs within medical training: (1) results from this survey provide information regarding harassment behavior that subjectively impacts a trainees' ability to perform their job but is not considered severe enough to report. Improved and targeted training programs can use this information to prepare our colleagues and trainees to directly address sexual harassment in the moment, particularly by patients; (2) improved and more transparent reporting mechanisms for inappropriate behavior are needed within our academic institutions and 
training programs. Current trainees report duty hours and rate the effectiveness of their program's learning environment (e.g., to the Accreditation Council for Graduate Medical Education); thus, training programs and accreditation bodies could use this standardized survey to identify high levels of harassment and/or hostile departmental culture that would warrant more significant institutional intervention.

\section{Limitations}

This study has several limitations. First, the initial sexual harassment comments were submitted only by female ophthalmology residents and faculty and ranked by female residents only. These comments represent a breadth of verbal harassment toward women ophthalmologists with varied years of experience. This study demonstrated a greater prevalence of sexual harassment toward male trainees than was previously reported, and future studies would benefit from inclusion of the male experience. Scoring of harassment comments was completed by female residents only, as the scale was designed to assess the experience of trainees. Other studies have suggested that faculty experience of harassment and response to harassment behavior is different from trainees. ${ }^{9}$

Surveying physicians has innate potential biases, and it is possible that those who responded do not accurately represent all United States ophthalmology trainees. Further, the true response rate is unknown as the survey was not distributed by all residency directors to their trainees. There was also no means to retrospectively contact program directors to determine who distributed the survey link without compromising anonymity. Trainees at programs whose directors have an interest in addressing sexual harassment may have been more likely to receive the survey. Limitations also include nonresponse bias, which could inflate estimates of prevalence if trainees who experienced harassment were more motivated to respond. The verbal sexual harassment experienced by trainees could be more frequent than reported given the survey did not inquire about experiences occurring more frequently than weekly. Further, these results were based on survey data and not documented cases. Future studies are indicated to determine more accurate rates of harassment.

\section{Conclusion}

This study should increase awareness of the high frequency at which sexual harassment impacts ophthalmology train- ees. There is a clear unmet need for comprehensive harassment training programs that empower physicians to respond to less severe harassment in the moment. Institutional cultures that create barriers to discussion and reporting must be examined and changed. Sexual harassment impacts all of us when it prevents the future leaders of our profession from performing at their highest potential. We owe it to our patients and to ourselves to take action.

\section{Conflict of Interest \\ None declared.}

\section{Acknowledgments}

The authors acknowledge the support of an unrestricted grant to the University of Iowa Department of Ophthalmology and Visual Sciences from Research to Prevent Blindness, Inc., New York, NY. There are no financial disclosures for any author.

\section{References}

1 Jagsi R, Griffith KA, Jones R, Perumalswami CR, Ubel P, Stewart A. Sexual harassment and discrimination experiences of academic medical faculty. JAMA 2016;315(19):2120-2121

2 Jagsi R. Sexual harassment in medicine - \#MeToo. N Engl J Med 2018;378(03):209-211

3 Cabrera MT, Enyedi LB, Ding L, MacDonald SM. Sexual harassment in ophthalmology: a survey study. Ophthalmology 2019;126(01): $172-174$

4 Lui PP, Quezada L. Associations between microaggression and adjustment outcomes: a meta-analytic and narrative review. Psychol Bull 2019;145(01):45-78

5 Thurston RC, Chang Y, Matthews KA, von Känel R, Koenen K. Association of sexual harassment and sexual assault with midlife women's mental and physical health. JAMA Intern Med 2019;179 (01):48-53

6 Till FJ. Sexual Harassment. A Report on the Sexual Harassment of Students. Washington, D.C.: National Advisory Council on Women's Educational Programs; 1980

7 Preventing Sexual Harassment. The U.S. Equal Employment Opportunity Commission Manual, BNA Communications, Inc. SDC IP.73; 1992

8 Fnais N, Soobiah C, Chen MH, et al. Harassment and discrimination in medical training: a systematic review and meta-analysis. Acad Med 2014;89(05):817-827

9 Cambier Z, Boissonnault JS, Hetzel SJ, Plack MM. Physical therapist, physical therapist assistant, and student response to inappropriate patient sexual behavior: results of a national survey. Phys Ther 2018;98(09):804-814 